\title{
Production cross sections of thulium radioisotopes for alpha-particle induced reactions on holmium
}

\author{
Ahmed Rufai Usman ${ }^{\mathrm{a}}$, Mayeen Uddin Khandaker ${ }^{\mathrm{b}, *}$, Hiromitsu Haba ${ }^{\mathrm{c}}$, Naohiko Otuka ${ }^{\mathrm{d}}$, \\ Masashi Murakami ${ }^{\mathrm{c}}$ \\ ${ }^{a}$ Department of Physics, Umaru Musa Yar'adua University, Katsina State, Nigeria \\ ${ }^{\mathrm{b}}$ Center for Biomedical Physics, School of Healthcare and Medical Sciences, Sunway University, 47500 Bandar Sunway, Selangor, Malaysia \\ ${ }^{\mathrm{c}}$ Nishina Center for Accelerator-Based Science, RIKEN, Wako, Saitama 351-0198, Japan \\ ${ }^{\mathrm{d}}$ Nuclear Data Section, Division of Physical and Chemical Sciences, Department of Nuclear Sciences and Applications, International Atomic Energy Agency, A-1400 \\ Vienna, Austria
}

A R T I C L E I N F O

\section{Keywords:}

Holmium

$(\alpha, \mathrm{x})$ reactions

${ }^{165-168} \mathrm{Tm}$ radioisotopes

Excitation functions

Thick target yields

\begin{abstract}
A B S T R A C T
The production cross sections of ${ }^{165-168} \mathrm{Tm}$ radioisotopes produced in $\alpha$-particle induced reactions on ${ }^{165} \mathrm{Ho}$ were measured from $49.6 \mathrm{MeV}$ down to the respective thresholds using the stacked-foil activation technique and HPGe gamma-ray spectrometry. The measured excitation functions were compared with previous measurements as well as the TENDL-2019 library. We also calculated the thick target yields for all the assessed radioisotopes by integration of the measured excitation functions and found that they show good agreement with the directly measured thick target yields except for ${ }^{168} \mathrm{Tm}$ production.
\end{abstract}

\section{Introduction}

There has been recent increase in labelling biomolecules with radioisotopes produced from rare earth metals for therapeutic applications $[1,2]$. Several radioisotopes produced from rare earth metals are pure emitters of Auger electrons, which could transfer large local doses to tissues and are thus coupled on biomolecules as a chelate as in DOTA for cancer treatment [3].

The $(n, \gamma)$ reactions are currently the most predominant production routes of therapeutic radioisotopes. However, charged-particle-induced nuclear reactions serve as good alternatives to produce these radioisotopes with high specific activities. Irradiation of holmium (Ho) with alpha particle beams leads to the production of several important thulium (Tm) radioisotopes for applications in nuclear medicine.

${ }^{167} \mathrm{Tm}$ was proposed as a useful radioisotope for studying bone and tumours $[4,5]$, while Chandra et al. showed that it could also be used as a skeletal imaging agent [4]. Low energy $\gamma$ - and X-rays emitted from the moderately long-lived ${ }^{167} \mathrm{Tm}$ (9.25 days) are suitable for radiotherapy $[1,6]$, making it effective in treating relatively small tumours which weigh $1 \mathrm{mg}$ or less [2]. On the other hand, ${ }^{165} \mathrm{Tm}\left(T_{1 / 2}=1.25 \mathrm{~d}\right)$ has been considered as a potential replacement of the popular ${ }^{167} \mathrm{Tm}$ [7] due to its desirable decay characteristics. ${ }^{167} \mathrm{Tm}$ can be produced in direct and indirect charged-particle induced reactions. Table 1 presents the major production routes for ${ }^{167} \mathrm{Tm}$ via the charged-particle-induced nuclear reactions $[2,6]$.

Aside from the medical applications, measurements of low energy nuclear processes via the $(\alpha, n)$ and $(\alpha, \gamma)$ reactions are very important in $\gamma$-process (p-process) nucleosynthesis, the photodisintegration phenomenon of nuclei heavier than Fe in explosive stellar processes, and to improve the determination of certain astrophysical reaction rates [8].

A comprehensive analysis of all previous measurements of alphaparticle induced reactions on holmium [6,9-19] shows large and unacceptable discrepancies. New measurements have therefore been made, using relatively large number of holmium foils, to explore the excitation functions at various energy points.

\section{Materials and method}

The overall procedure employed in the present work is similar to our previous studies [20-23]. The stacked target foils were irradiated with a beam of alpha-particles, and the foils were subjected to the gamma-ray spectrometry to determine the production cross sections.

\subsection{Targets and irradiation}

Thin metallic holmium foils (purity: 99\%, thickness: $12.29 \mu \mathrm{m}$, supplier: Goodfellow, UK) served as the target. There is only one stable isotope, ${ }^{165} \mathrm{Ho}(100 \%)$, in natural holmium [24]. Natural copper foils

\footnotetext{
* Corresponding author.

E-mail address: mayeenk@sunway.edu.my (M.U. Khandaker).
} 